\title{
Formation and control of electron molecules in artificial atoms: Impurity and magnetic-field effects
}

\author{
Constantine Yannouleas and Uzi Landman \\ School of Physics, Georgia Institute of Technology, Atlanta, Georgia 30332-0430
}

(To appear in Phys. Rev. B 61 [15 June 2000])

\begin{abstract}
Interelectron interactions and correlations in quantum dots can lead to spontaneous symmetry breaking of the self-consistent mean field resulting in formation of Wigner molecules. With the use of spin-and-space unrestricted Hartree-Fock (sS-UHF) calculations, such symmetry breaking is discussed for field-free conditions, as well as under the influence of an external magnetic field. Using as paradigms impurity-doped (as well as the limiting case of clean) twoelectron quantum dots (which are analogs to helium-like atoms), it is shown that the interplay between the interelectron repulsion and the electronic zero-point kinetic energy leads, for a broad range of impurity parameters, to formation of a singlet ground-state electron molecule, reminiscent of the molecular picture of doubly-excited helium. Comparative analysis of the conditional probability distributions for the sS-UHF and the exact solutions for the ground state of two interacting electrons in a clean parabolic quantum dot reveals that both of them describe formation of an electron molecule with similar characteristics. The self-consistent field associated with the triplet excited state of the two-electron quantum dot (clean as well as impurity-doped) exhibits symmetry breaking of the Jahn-Teller type, similar to that underlying formation of nonspherical open-shell nuclei and metal clusters. Furthermore, impurity and/or magnetic-field effects can be used to achieve controlled manipulation of the formation and pinning of the discrete orientations of the Wigner molecules. Impurity effects are futher illustrated for the case of a quantum dot with more than two electrons.
\end{abstract}

Pacs Numbers: 73.20.Dx, 71.45.Lr, 73.23.-b

\section{INTRODUCTION}

Two-dimensional (2D) quantum dots (QD's) created at semiconductor interfaces with refined control of their size, shape and number of electrons are often referred 13 to as "artificial atoms". This analogy suggests that the physics of electrons in such man-made nanostructures is closely related to that underlying the traditional description 1 of natural atoms (pertaining particularly to electronic shells and the Aufbau principle), where the electrons are taken $\overline{5}$ to be moving in a spherically averaged effective central mean field (CMF). However, using as paradigms impurity-doped (as well as the limiting case of clean) twoelectron-QD $(2 e \mathrm{QD})$ analogs to He-like atoms, we show that the interplay between the interelectron repulsion
$(Q)$ and the electronic zero-point kinetic energy $(K)$ may lead, for a broad range of impurity parameters, to spontaneous symmetry-breaking (SB) of the self-consistent mean field, resulting at zero magnetic field $(B=0)$ in formation of a singlet ground-state electron molecule. Such $\mathrm{SB}$ is beyond the CMF picture and, while negligible in the ground state of the He atom (whose study was central to the development of the quantum theory of matter due to the failure of the Bohr-type models 6 ), it is similar in nature to the SB found 6 in the 1970's in doubly excited $\mathrm{He}$, where formation of an $e-\mathrm{He}^{2+}-e$ "triatomic" molecule has been invoked. Furthermore, we show that symmetry breaking at $B=0$ of the self-consistent field associated with the triplet excited state of the $2 e \mathrm{QD}$ originates from a Jahn-Teller distortion of the CMF, similar to that underlying formation f nonspherical open-shell nucleit, 8 and metal clusters. 10

Along with a unification of concepts pertaining to spontaneous SB in a variety of finite fermionic systems (from nuclei, metal clusters and natural atoms, to $2 \mathrm{D}$ QD's), we demonstrate the ability to control the orientation and to manipulate (i.e., to enhance, but also to counteract and even to void) the formation of the electron molecules in 2e QD's via impurity and/or magnetic-field effects. The ability to control the orientation of the electron molecule may in principle open new possibilities for designing "on-off" (i.e., switching between two discrete states) devices, which eventually may be employed in applications of QD's as nanoscale logic gates11 (the effect of impurities on the structure of multi-electron molecules in QD's with more than two electrons is further illustrated in Appendix A).

That electrons in extended media may undergo crystallization at low densities, when $Q$ dominates over $K$, has been predicted 12 by Wigner in 1934. Such Wigner crystallization (WC) in clean QD's results in formation of electron molecules 13 [16 [also referred to as Wigner molecules (WM's)], which are associated with spontaneous SB, where the symmetry of the ground state, calculated at the mean-field (self-consistent-potential) level, is found to be lower than that of the exact hamiltonian describing the system 1719 In clean QD's, formation 20 of WM's is controlled 1621 by the parameter $R_{W}=Q / K$. For a parabolic confinement (with frequency $\omega_{0}$ ) at $B=$ 0 , it is customary to take $Q=e^{2} / \kappa l_{0}$ and $K \equiv \hbar \omega_{0}$, where $\kappa$ is the dielectric constant and $l_{0}=\left(\hbar / m^{*} \omega_{0}\right)^{1 / 2}$ is the spatial extent of the lowest state's wave function of an electron with an effective mass $m^{*}$; WM's occur 16 for $R_{W}>1$, corresponding to much higher electron 
densities 15,16 than those predicted for WC in an infinite 2D medium.22

The many-body hamiltonian for a QD with $N_{e}$ electrons can be expressed as a sum of a single-particle part and the two-particle interelectron Coulomb repulsion,

$$
\mathcal{H}=\sum_{i=1}^{N_{e}} H(i)+\sum_{i=1}^{N_{e}} \sum_{j>i}^{N_{e}} \frac{e^{2}}{\kappa r_{i j}}
$$

The contributions to the single-particle part are written as

$$
H(i)=H_{0}(i)+H_{B}(i)+V_{I}(i),
$$

and they contain a term describing the motion of an electron in a $2 \mathrm{D}$ parabolic confinement, i.e., $H_{0}(i)=$ $\mathbf{p}_{i}^{2} / 2 m^{*}+m^{*} \omega_{0}^{2}\left(x_{i}^{2}+y_{i}^{2}\right) / 2$, where $\omega_{0}$ is the frequency of the $2 \mathrm{D}$ isotropic harmonic confining potential. Magneticfield effects are included in $H_{B}(i)=\left[\left(\mathbf{p}_{i}-e \mathbf{A}_{i} / c\right)^{2}-\right.$ $\left.\mathbf{p}_{i}^{2}\right] / 2 m^{*}+g^{*} \mu_{B} \mathbf{B} \cdot \mathbf{S}_{i} / \hbar$, where the vector potential, $\mathbf{A}_{i}=$ $B\left(-y_{i} / 2, x_{i} / 2,0\right)$, is taken in the symmetric gauge, and the last term is the Zeeman interaction with an effective factor $g^{*}, \mathbf{S}_{i}$ is the electron spin, and $\mu_{B}$ the Bohr magneton. To include the effect of (Coulombic) impurities, we added to $H(i)$ the term $V_{I}(i)=(e / \kappa) \sum_{l} \mathcal{Q}_{l} /\left|\mathbf{r}_{i}-\mathcal{R}_{l}\right|$ $\left(i=1, \ldots, N_{e}\right)$, where $\mathcal{Q}_{l}$ is the charge of the $l$ th impurity located at $\mathcal{R}_{l}=\left(x_{l}, y_{l}, d_{l}\right)$; such impurities which in general may be situated out of the $2 \mathrm{D}$ plane of the QD (that is $d_{l} \neq 0$ ) may correspond to implanted atoms (donors or acceptors) or represent a fabricated, controllable voltagegate.

A clean QD (that is with $\mathcal{Q}_{l}=0$ in the above hamiltonian) may be regarded as a realization of the Thomson atom, as are jellium models of metal clusters, 10 where the positive charge is uniformly distributed; in the $2 e$ Thomson QD (TQD), the confinement to the 2D plane is modeled by a parabolic potential. On the other hand, a QD analog of the Rutherford model of the atom (RQD), where the positive charges are grouped in a single nucleus, can be achieved through the introduction of a central attractive impurity (in addition to the harmonic confinement).

The presence of many free parameters in Eqs. (11) and (2) results in a plethora of possible case studies generated by varying the material dependent parameters $(\kappa$ and $\left.m^{*}\right)$, the harmonic confinement $\omega_{0}$ and the magnetic-field $B$, as well as the number of impurities $l$, their charges $\mathcal{Q}_{l}$, and positions $\mathcal{R}_{l}$. In this paper, we have chosen to discuss here the following three representative classes of cases at a specific value of $R_{W}=2.39$, which is sufficientlly high so that the electrons form 16 a WM in the case of a clean QD, thus allowing for systematic investigations of the effects of impurities on the formation, orientational pinning, and structural distortions of the electron molecules (for the other parameter values used throughout this paper, see Ref.23). The three representative classes, which we discuss, are: (i) A $2 \mathrm{e}$ TQD at $B=0$ in the presence of two off-centered impurities situated on both sides of the dot (section II); (ii) A 2e RQD (with a single attractive central impurity) in the presence of an applied magnetic field (for both the cases of a weak and a strong field, section III); and (iii) A QD with eight electrons at $B=0$ and a single impurity with varying strength, polarity, and location (Appendix A).

For the case of a clean 2e QD, with the hamiltonian given by Eqs. (11) and (2) with $N_{e}=2$ and $V_{I}=0$, the exact solution can be found quite easily 2428 owing to the separability of the Schrödinger equation in the center-ofmass and relative coordinates; in the presence of impurities, separability is lost and finding an exact solution 29 becomes significantly more complicated (even for two electrons in the presence of a central impurity). Therefore, and also in order to expound the physical principles underlying spontaneous symmetry breaking in QD's, we will mostly use in the following (sections II and III and Appendix A) the self-consistent spin-ayd-space unrestricted Hartree-Fock (sS-UHF) method 30 which, unlike the restricted $\mathrm{HF}(\mathrm{RHF})$ technique 31,32 allows for the formation of broken-symmetry states (of lower energy than those obtained via the RHF). This sS-UHF, which we introduced for studies of QD's in Ref. 16, employs $N_{e}$ (where $N_{e}$ is the number of electrons) orbitaldependent, effective (mean-field) potentials and it differs from the usual 33 RHF in two ways: (i) it relaxes the double-occupancy requirement, namely, it employs different spatial orbitals for the two different (i.e., the up and down) spin directions [DODS, thus the designation "spin (s) unresricted"], and (ii) it relaxes the requirement that the electron orbitals be constrained by the symmetry of the external confining field [thus the designation "space (S) unrestricted"].

Subsequent to our discussion of symmetry breaking and formation of electron molecules in the framework of the sS-UHF method, we elaborate in section IV on the connection between the symmetry-broken sS-UHF solution and the exact one in the case of a clean $2 \mathrm{e}$ QD with $B=0$. In particular, analysis of the exact solution using the conditional-probability-distribution (CPD) technique 34 reveals the formation of an electron molecule in agreement with the sS-UHF result.

\section{THOMSON QUANTUM DOT WITH OUTSIDE IMPURITIES}

To introduce some of the principal physical and methodological issues pertaining to symmetry breaking and formation of Wigner molecules in finite fermion systems, we discuss first the $2 e$ TQD at $B=0$ [we remind the reader that a sufficiently high value of $R_{W}(=2.39)$ was chosen, such that the QD is in the regime where the two electrons form a WM; for the other parameters used throughout this paper, see Ref. 23. The single-particle wave functions (modulus square) and total electron densities displayed in Fig. 1 are taken from calculations for 
the $2 e$ TQD in the presence of two attractive impurities $\left(\mathcal{Q}_{1}=\mathcal{Q}_{2}=-1 e\right.$, represented in the figure by filled dots on the two sides of the QD) located symmetrically about the center of the QD at $(x, y, z)=( \pm 60,0,10) \mathrm{nm}$ with the strength and location of the impurities purposefully chosen such that they will not affect the nature of the electronic ground states (for the same QD but without the impurities), except for orientational pinning in the case of symmetry-broken states (see below).

Constraining first the solution to maintain the symmetry of the hamiltonian, in conjunction with doubleoccupancy of the HF orbitals by electrons of opposite spins [that is through the use of the RHF method with input trial electron densities satisfying the symmetry of the external potentials 31 , the resulting symmetry-adapted $(\mathrm{SA})$ self-consistent singlet $(\mathcal{S})$ orbitals and corresponding total density distributions exhibit, as expected, an almost circular symmetry with minimal elliptical distortions (due to the impurities), see Fig. 1(a); without the impurities, the SA singlet is perfectly circularly symmetric.

However, increasing the variational freedom through removal of the spatial symmetry and double-occupancy constraints via the use of the SS-UHF method results in a symmetry-broken singlet ground state of lower energy, that is formation of a WM characterized by localized orbitals with the "bond length" (distance between the maxima in the total electronic distribution) equal to $29 \mathrm{~nm}$ [see Fig. 1(b)]; the energy of this state is lower by $1.62 \mathrm{meV}$ than that of the SA solution [Fig. 1(a)]. This lowering of the ground-state energy reflects gain in correlation energy (for the definition of correlation energy, see section IV below). Note that the 2e WM is orientationally pinned along the interimpurity axis. Similar formation of a WM occurs also for a $2 e$ TQD without the pinning impurities (with an energy gain of $1.32 \mathrm{meV}$ compared to the corresponding SA solution). However, in the absence of pinning, the formation of the WM is accompanied by orientational degeneracy 35 (that is there is an infinite manifold of rotationally degenerate sS-UHF ground states).

The formation of a fermionic molecule, associated with electron localization, in the ground-state of a QD under magnetic-field-free conditions does not have an analog within the framework of the traditional models of atomic structure. 1 However, the physics underlying this phenomenon, which is a manifestation of SB resulting from strong electronic correlations (see also section IV below), resembles closely that found in doublyexcited two-electron atoms. Indeed, spectroscopical studies on doubly-excited helium atoms revealed rovibrational bands which were interpreted, borrowing from models developed in the context of nuclear and molecular physics, by invoking the formation of a "triatomic" molecule comprised of the two localized electrons and the $\mathrm{He}^{2+}$ nucleus ( $\alpha$ particle), with the collinear configuration being of particular significance.6

The first electronically excited state of the $2 e$ TQD is the triplet $\mathcal{T}$ state (with the spins of the two electrons parallel to each other) whose total electron density distribution [Fig. 1(c), left] resembles that of the groundstate singlet [Fig. 1(b), left]. However, the individual electronic wave functions in the $\mathcal{T}$ state differ in character from those of the $\mathcal{S}$ state [compare right panels in Fig. 1(c) and 1(b)], with the lower-energy one being $s$ like (but elliptically distorted), and the other is a $p$-like orbital oriented by the impurities along the $x$-axis. Note that the $\mathcal{T}$ state has the symmetry of the hamiltonian including the two pinning impurities (that is, here the sS-UHF solution coincides with the symmetry-adapted one). The same orbital characters are obtained also in the absence of the pinning impurities, but without a preferred orientation. In this case, however, the character of the $\mathcal{T}$ state is a result of spontaneous SB, with an energy gain of $0.09 \mathrm{meV}$ compared to the corresponding SA (circular) solution. Underlying the type of spontaneous SB in the (open-shell $\mathcal{T}$ state of the $2 e$ TQD is the JahnTeller (JT) effect 36 where lowering of the total energy is achieved via mixing of the two-fold degenerate $m=+1$ $\left(p_{+}\right)$amd $m=-1\left(p_{-}\right)$angular momentum states, concomitant with a deformation of the self-consistent potential away from circular symmetry 37 To distinguish such electron molecules from the WM discussed above for the closed-shell singlet state (whose formation is driven by the dominance of the electron-electron repulsion), we refer to them as JT electron molecules (JTEM's). Such spontaneous SB via JT distortions is familiar from studies of the rotational spectra of open-shell nucleid 8 and from investigatipns of shape deformations of open-shell metal clusters. 0.10

Similar calculations for the $2 e$ TQD, but with repulsive pinning impurities (that is $\mathcal{Q}_{1}=\mathcal{Q}_{2}=+1 e$ ) yield for the singlet ground state qualitatively similar results (with different values for the energies), but with an important distinction that now the pinned orientation of the $\mathrm{WM}$ is rotated by $\pi / 2$ compared to the $\mathcal{Q}_{1}=\mathcal{Q}_{2}=-1 e$ case (i.e., the "intramolecular" axis of the WM is oriented normal to the interimpurity axis). Consequently, through variation of the sign (polarity) of the impurity gate voltages, one may "flip" the orientation of the WM, and with it the direction of the polarization of the electronic charge distribution in the QD. In this way, the formation of WM's in QD's and the ability to control their discrete orientations via pinning voltage gates may serve as a method for the creation of on-off information storage cells and nanoscale logic gates. 11

\section{RUTHERFORD QUANTUM DOT}

Next, we examine the properties of a $2 e$ Rutherford $\mathrm{QD}(\mathrm{RQD})$, that is a $2 e \mathrm{QD}$ with a central attractive impurity. The sS-UHF singlet and triplet electronic orbitals corresponding to a $2 e \mathrm{RQD}$ for $B=0$ with a single impurity $(\mathcal{Q}=-2 e)$ located at $(0,0,10) \mathrm{nm}$ are shown in Fig. 
2(b) and 2(c) respectively. They exhibit WM symmetry breaking and JT-distortion features similar to those found for the 2e TQD [compare Fig. 1(b and c)], but with a reduced WM bond length and a more compact triplet. The "strength" of the SB depends of course on the impurity charge $\mathcal{Q}$ and/or its distance $d$ from the QD plane. For example, for $\mathcal{Q}=-1 e$ and $d=0$ (and for an arbitary position of the impurity inside the QD), no symmetry breaking was found by us due to the strong trapping by the impurity of the two electrons which occupy circularly symmetric orbitals, resembling the behavior of the ground-state of the natural He atom. 38 We also remark that for the case described in Fig. 2(b) and Fig. 2(c) both electrons are slightly trapped by the impurity potential for the $\mathcal{S}$ state, while for the $\mathcal{T}$ state the $s$-like electron is strongly trapped and the $p$-like electron occupies a much less bound orbital.

Note that here, as with the case of a clean QD, the singlet WM and the JT-distorted triplet are free to rotate in the plane of the $\mathrm{QD}$, since there are no off-centered pinning impurities.

The large physical size of QD's makes them ideally suited for investigations of magnetic-field effects and controlled manipulations. To illustrate such effects, we display in Fig. 2(a) the magnetic-field induced variation of the total energies of the $\mathcal{S}$ and $\mathcal{T}$ states in the 2e RQD (qualitatively similar behavior is found also for the $2 e$ TQD). As seen, the energy of the singlet state increases and that of the triplet state decreases with increasing $B$; for fields $B<B_{1}(0.2 \mathrm{~T})$, the variation of the energy of the $\mathcal{T}$ (the slope of the curve) is smaller than that for $B>B_{1}$ [see inset in Fig. 2(a)]. Furthermore, at a critical value $B_{c}=2.8 \mathrm{~T}$ [marked by a down-arrow in Fig. 2(a)], the energies of the two states cross and from then on the triplet lies below the singlet.

For the singlet, the broken-symmetry WM state [Fig. 2(b)] maintains under the influence of the applied magnetic field in the range considered in Fig. 2(a), with the increase in the magnetic-field strength leading to further shrinkage of the bond length of the WM accompanied by an overall increase of the energy of the $\mathcal{S}$ state 39 [see Fig. $2(\mathrm{a})]$.

The influence of the magnetic field on the triplet state is more subtle. As aforementioned, at $B=0$ the symmetry of the $\mathcal{T}$ state is broken by the JT effect involving mixing of the $m= \pm 1$ degenerate angular momentum orbitals (see Ref. 37). On the other hand, the magnetic field lifts the degeneracy of these $p_{+}$and $p_{-}$states (without mixing them), and this effect competes with the JT distortion. For small enough fields $\left[B<B_{1}\right.$, see inset in Fig. 2(a)], the JT effect prevails, and thus the orbitals and electron densities remain similar to those shown in Fig. 2(c), and they maintain an orientational degeneracy in the plane. At stronger fields $\left(B>B_{1}\right)$, the lifting of the energetic degeneracy of the $p_{+}$and $p_{-}$states overcomes the JT effect, and the second electron populates the lower of these two orbitals. As a result, circular symmetry is recovered, as illustrated in Fig. 2(d).
The overall decrease with $B$ of the energy of the $\mathcal{T}$ state relative to the $\mathcal{S}$ state is due to enhanced stabilization by the (parallel-spin) exchange energy in the former, reduced Coulomb repulsion between the electrons occupying $s$ - and $p$-like orbitals, and quenching of the kinetic energy of the $p$-like orbital by the magnetic field. 39 This effect increases with $B$, and at $B \geq B_{c}$ the $\mathcal{T}$ state becomes the ground state [see Fig. 2(a)]. Note that this transition is driven primarily by the interelectron repulsion and not by the interaction of the electrons' moments with the magnetic field (see Ref. 2 and references therein); for our system, the Zeeman splitting energy is $0.026 \mathrm{meV} / \mathrm{T}$. At even larger fields, the Coulomb repulsion between the electrons increases (due to the shrinking of the orbitals) resulting in an ascending trend of the energy of the $\mathcal{T}$ state, which remains, however, lower than the singlet state. A similar scenario is found also for the TQD (without pinning impurities)

In light of previous findings 13.42 pertaining to formation of fully spin-polarized symmetry-broken states in clean QD's (TQD's) at high magnetic fields, it is pertinent to inquire whether the circular symmetry found for the $\mathcal{T}$ state of the $2 e \mathrm{RQD}$ for $B_{1}<B<5 \mathrm{~T}$ will also be broken at higher fields. For the clean $2 e \mathrm{TQD}$, we verified that indeed an orientationally degenerate electron molecule [with the molecular orbitals of the electrons distributed about the two molecular centers, see Fig. 2(e)] formed at sufficiently high $B$ (e.g., $B=10 \mathrm{~T}$ ). Such an electron molecule is akin 40 to the JTEM discussed above in the context of the triplet state for $B=0$. Interestingly, such reemergence of a JTEM structure does not occur at these conditions for the $2 e$ RQD studied here due to the enhanced gap between the $p_{+}$orbital and the strongly trapped $s$ orbital. This provides an additional venue for impurity-assisted manipulation and design of the electronic properties of QD's.

\section{CONNECTION TO THE EXACT SOLUTION}

As mentioned earlier, for $R_{W}>1$, the sS-UHF approach applied to QD's yields approximate ground-state solutions which violate the symmetries of the original many-body hamiltonian, e.g, the spontaneous breaking of rotational symmetry discussed in sections II and III for a circularly symmetric clean QD (i.e., a TQD) or one with a central impurity (i.e., RQD). At a first glance, this situation may appear puzzling, but it is not unique in the context of many-body theory of finite fermionic systems. Indeed, a similar situation was encountered in nuclear physics in the 1950's, when it was discovered that open shell nuclei carried permanent quadrupole moments and that many of them exhibited well developed rotational spectra (i.e., they behaved like rigid rotors). The explanation of these experimental findings was formulated in the framework of breaking of the rotational symmetry associated with nuclear deformations 
of the Jahn-Teller type, and it led to several celebrated models and semi-empirical methods, i. en the particleplus-rotor model of Bohr and Mottelspy, 41 the modifiedanisotropic-oscillator model of Nilsson 42 and the Strutinsky shell-correction method. 43 In the language used by us here, this means that at the microscopic level the breaking of the rotational symmetry had to be accounted 44 for via space (S)-UHF methods (that is allowing the spatialorbital solutions of the Hartree-Fock equations to assume symmetries lower than those of the underlying manybody hamiltonian).

Starting with Peierls and Yocco 45 (see also Peierls and Thoules.46), numerous theoretical investigationss have addressed the connection of the broken-symmetry HF solution to the exact solution, and led to the theofy of restoration-of-symmetry via Projection Techniques. 17 The central physical ingredient of the Projection Technique rests with the observation that a $\mathrm{HF}$ solution which breaks rotational symmetry is not unique, but belongs to an infinitely-degenerate manifold of states with different spatial orientation. A proper linear combination of the HF determinants in such a manifold yields multideterminental states with good total angular momenta that are a better approximation to the exact solution. For a comprehensive review on restoration of symmetry in the context of nuclear many-body theory, we refer the reader to the book by Ring and Schuck mentioned in Ref. 35 (see also Ref. 48, where the principles of restoration of rotational symmetry are discussed in the two-dimensional case).

The restoration of symmetry via Projection Techniques in the case of sS-UHF solutions describing 2D-QD's will be presented in a future publication. In this section, we found it more convenient to discuss the connection between the sS-UHF and the exact solutions by taking advantage of the simplicity of solving the exact problem at $B=0$ for two electrons interacting via the Coulomb force and confined by an external parabolic confinement without impurities (clean QD). Indeed, it is well known that in this case, the exact Schrödinger equation for two interacting electrons is separable in the center-of-mass, $\mathbf{R}=\left(\mathbf{r}_{1}+\mathbf{r}_{2}\right) / 2$ (with a corresponding mass $\mathcal{M}=2 m^{*}$ ), and relative, $\mathbf{r}=\mathbf{r}_{1}-\mathbf{r}_{2}$ (with a corresponding reduced mass $\left.\mu=m^{*} / 2\right)$, coordinates.

To analyze the properties of the exact solutions, two quantities 25.27 have customarily been extracted from the two-body wave function $\Psi\left(\widetilde{\mathbf{r}}_{1}, \widetilde{\mathbf{r}}_{2}\right)$ [where the tilde denotes both spatial and spin variables, i.e., $\widetilde{\mathbf{r}}_{i} \equiv\left(\mathbf{r}_{i}, s_{i}\right)$ $(i=1,2)$ see Appendix B]: (i) the usual pair-correlation function 49

$$
G(v)=2 \pi\left\langle\Psi\left|\delta\left(\mathbf{r}_{1}-\mathbf{r}_{2}-\mathbf{v}\right)\right| \Psi\right\rangle
$$

and (ii) the electron density (ED)

$$
n(v)=\left\langle\Psi\left|\sum_{i=1}^{2} \delta\left(\mathbf{v}-\mathbf{r}_{i}\right)\right| \Psi\right\rangle .
$$

However, for the exact $\Psi$ in the case of a circularly symmetric confinement, both of these quantities turn out to be also circularly symmetric and thus they do not reveal the full physical picture, the (possible) formation of an electron molecule generated by electron correlations. A more suitable quantity here is the conditional probability distribution (CPD) $\mathcal{P}\left(\mathbf{v} \mid \mathbf{r}_{2}=\mathbf{v}_{0}\right)$ for finding one electron at $\mathbf{v}$ given that the second electron is at $\mathbf{r}_{2}=\mathbf{v}_{0}$. This quantity has been extensively used 34 in the analysis of electron correlations in doubly-excited helium-like atoms, and is defined as follows,

$$
\mathcal{P}\left(\mathbf{v} \mid \mathbf{r}_{2}=\mathbf{v}_{0}\right) \equiv \frac{\left\langle\Psi\left|\delta\left(\mathbf{v}-\mathbf{r}_{1}\right) \delta\left(\mathbf{v}_{0}-\mathbf{r}_{2}\right)\right| \Psi\right\rangle}{\left\langle\Psi\left|\delta\left(\mathbf{v}_{0}-\mathbf{r}_{2}\right)\right| \Psi\right\rangle}
$$

[for details concerning the calculation of the quantities in Eqs. (3) - (5), see Appendix B].

In the left column of Fig. 3, we display the above three quantities for the exact ground state in the case of a parabolic confinement with the same parameters as used throughout this paper (see Ref. 23) and $B=0$. The pair-correlation function in Fig. 3(a) exhibits a well developed depression at $v=0$, namely the two electrons on the average keep apart from each other at a distance $2 r_{0}=22.92 \mathrm{~nm}$. In Fig. 3(b), we plot the CPD with $\mathbf{v}_{0}=\left(r_{0}, 0\right)$ (marked by a cross). It is seen that the maximum probability for finding the second electron occurs at the diametrically opposite point $-\mathbf{v}_{0}$, in accordance with the picture of an electron molecule presented earlier within the sS-UHF approach. The exact electron density shown in Fig. 3(c) is circularly symmetric, as expected. Comparing figures 3(b) and 3(c), the following interpretation ensues naturally, namely that the CPD in Fig. 3(b) describes the electron molecule in its intrinsic (body-fixed) frame of reference, while the electron density in Fig. 3(c) describes the electron molecule in the laboratory frame of reference where rotational and and center-of-mass displacements are superimposed upon the intrinsic probability density.

Fig. 3(d) displays the electron density for the corresponding sS-UHF ground state. As discussed earlier, the sS-UHF electron density breaks the rotational symmetry and clearly exhibits the morphology of an electron molecule, unlike the exact one in Fig. 3(c). It is apparent that the $\mathrm{sS}-\mathrm{UHF}$ electron density corresponds to that in the intrinsic frame of the electron molecule. Restoration of the symmetry via Projection Techniques will bring the sS-UHF electron density closer to that of the exact solution. As aforementioned, this interpretation is familiar in the context of nuclear and it is further supported by the CPD calculated with the sS-UHF ground-state [i.e., by using $\Psi^{\mathrm{UHF}}$ in Eq. (5), instead of the exact many-body $\Psi$, see Appendix B] and displayed in Fig. 3(e). Although naturally not identical, the two CPD's (i.e., the exact and the UHF) are of similar nature and both illustrate graphically the correlation effect associated with electron localization and formation of an electron molecule. We further illustrate this point by contrasting the exact and 
sS-UHF CPD's with the CPD of the symmetry-adapted RHF ground state, shown in Fig. 3(f). In this latter case, $\Psi^{\mathrm{RHF}}=\psi^{\mathrm{RHF}}\left(\mathbf{r}_{1}\right) \psi^{\mathrm{RHF}}\left(\mathbf{r}_{2}\right) \chi\left(s_{1}, s_{2} ; S=0\right)$, with $\psi^{\mathrm{RHF}}(\mathbf{v})$ being the $1 s$ orbital of the RHF; as a result, the probability of finding one electron at $\mathbf{v}$ is independent of the position of the second electron and it is centered about the origin where it achieves its maximum value, as expected from an independent-particle description, i.e.,

$$
\mathcal{P}^{\mathrm{RHF}}\left(\mathbf{v} \mid \mathbf{r}_{2}=\mathbf{v}_{0}\right)=\left|\psi^{\mathrm{RHF}}(\mathbf{v})\right|^{2} .
$$

Finally it is of interest to examine the energetic aspects of the symmetry breaking. Indeed the energy of the ground-state is $E_{\mathrm{gs}}^{\mathrm{RHF}}=22.77 \mathrm{meV}$ for the RHF solution, $E_{\mathrm{gs}}^{\mathrm{UHF}}=21.45 \mathrm{meV}$ for the $\mathrm{sS}-\mathrm{UHF}$ solution, and $E_{\mathrm{gs}}^{\mathrm{ex}}=19.80 \mathrm{meV}$ for the exact one. Since the correlation energy is the difference between the RHF and the exact energies 50 one finds $\varepsilon_{\mathrm{gs}}^{\mathrm{corr}}=E_{\mathrm{gs}}^{\mathrm{RHF}}-E_{\mathrm{gs}}^{\mathrm{ex}}=2.97 \mathrm{meV}$. Thus the symmetry breaking associated with the sS-UHF solution is able to capture $\left(E_{\mathrm{gs}}^{\mathrm{RHF}}-E_{\mathrm{gs}}^{\mathrm{UHF}}\right) / \varepsilon_{\mathrm{gs}}^{\text {corr }}=44 \%$ of the correlation energy; the remaining amount can be captured through improvements via Projection techniques.11

\section{SUMMARY}

The dominance of interelectron interactions and correlations in quantum dots (often referred to as "artificial atoms") results in spontaneous symmetry breaking of the self-consistent mean field, beyond the centralfield picture on which the modern understanding of atomic structure has been founded. Indeed, as shown in this paper through spin-and-space unrestricted HartreeFock 16,30 many-body calculations for the singlet ground state of a 2e QD, such symmetry breaking and the resulting formation of Wigner molecules do occur for a wide range of system parameters (with or without impurities) even in the most elementary case of two-electron QD's.

Additionally, in the case of fully polarized dots (e.g., the triplet state of a 2e QD), the sS-UHF calculations show that similar electron molecules form due to symmetry breaking associated with Jahn-Teller-type distortions.

In spite of the simplicity of the hamiltonian, correlations in two-electron QD's underlie a remarkably rich and complex physical behavior. As a result, 2e QD's can serve as paradigms for a unification of concepts pertaining to spontaneous SB in various finite fermion aggregates, including nuclei, excited atoms, and clusters.

Furthermore, impurity and/or magnetic-field effects allow for controlled manipulation of the formation and pinning of the discrete orientations of the electron molecules in 2e QD's. Such ability may be employed in future applications of QD's as nanoscale logic cells and information storage elements. Impurity effects were also illustrated for the case of a quantum dot with more than two electrons.
Confirmation of the formation in 2e QD's of electron molecules associated with symmetry breaking of the selfconsistent field (in the context of SS-UHF calculations) was obtained through an analysis of the exact ground state via the conditional-probability-distribution technique.

\section{ACKNOWLEDGMENTS}

This research is supported by the US D.O.E. (Grant No. FG05-86ER-45234).

\section{APPENDIX A: QD'S WITH MORE THAN TWO ELECTRONS}

In the main body of this paper we limited ourselves to the case of 2e QD's, since focusing on this elementary case allowed us to better elucidate the intricate physical principles involved in the formation and impurity control of electron molecules in QD's, from both the perspective of the sS-UHF treatment and the exact solution. In this appendix, we will present an example of the many different structural possibilities that can arise when impurities are introduced in a QD with a larger number of electrons. In particular, we consider a QD with 8 electrons in the presence of a hydrogenic-like $(d=0)$ impurity of variable nominal charge $\mathcal{Q}=q e$ placed at the center (and in one case off-center) of the QD (for the other input parameters, we use same values as used throughout the text, see Ref. 23). Since this appendix does not intend to present an exhaustive study of larger dots, but simply aims at presenting an illustrative example, we will consider only one spin configuration, i.e., the sS-UHF solutions having 4 spin-up and 4 spin-down electrons. However, $q$ will be allowed to take both positive (repulsive) and negative (attractive) values.

Fig. 4(a) displays the sS-UHF electron density for a repulsive central impurity [located at $(0,0,0)$ ] with $\mathcal{Q}=+1 e$. It is seen that a Wigner molecule consisting of a single ring with 8 electrons [denoted as a $(0,8)$ ring] is formed in this case. For a slightly attractive central impurity with $\mathcal{Q}=-0.2 e$, however, a structural change takes place, namely one electron moves to the center of the dot [see Fig. 4(b)]. Notice that this $(1,7)$ structure agrees with the arrangement found in studies of classical point-charges in a purely $(\mathcal{Q}=0)$ harmonic confinement 20 Increasing the attractive nominal charge to $\mathcal{Q}=-0.5 e$ [see Fig. 4(c)] results in a further structural change, namely the central impurity is now able to trap two electrons, thus leading to a $(2,6)$ arrangement. A further increase of the attractive charge of the impurity to the value $\mathcal{Q}=-1 e$ does not produce any qualitative change in the $(2,6)$ arrangement as long as the impurity remains at the center of the dot. By moving the impurity to an off-center position, however, various structural 
morphologies can arise, an example of which is presented in Fig. 4(d) for a $\mathcal{Q}=-1 e$ impurity located at $(20,0,0)$ $\mathrm{nm}$, forming a highly distorted $(2,6) \mathrm{WM}$ (here the first index denotes that two electrons are trapped by the impurity).

A further increase in the attractive charge $\mathcal{Q}$ leads to sequential trapping of the remaining six electrons and to progressive elimination of symmetry breaking, until all eight electrons have been captured by the impurity (see also section III).

\section{APPENDIX B: TWO-ELECTRON WAVE FUNCTIONS AND THE DEFINITIONS (3)-(5)}

\section{Exact solution for two electrons}

In the case of two interacting electrons confined by a parabolic potential (clean QD), one can perform a change of variables to center-of-mass $(\mathrm{CM}), \mathbf{R}=\left(\mathbf{r}_{1}+\mathbf{r}_{2}\right) / 2$ and $\mathbf{P}=\mathbf{p}_{1}+\mathbf{p}_{2}$, and relative-motion $(\mathrm{rm}), \mathbf{r}=\mathbf{r}_{1}-\mathbf{r}_{2}$ and $\mathbf{p}=\left(\mathbf{p}_{1}-\mathbf{p}_{2}\right) / 2$, coordinates. Then the two-electron hamiltonian separates into $\mathrm{CM}$ and rm contributions,

$$
\mathcal{H}=\mathcal{H}_{\mathrm{CM}}+\mathcal{H}_{\mathrm{rm}},
$$

with

$$
\mathcal{H}_{\mathrm{CM}}=\frac{\mathbf{P}^{2}}{2 \mathcal{M}}+\frac{1}{2} \mathcal{M} \omega_{0}^{2} R^{2}
$$

and

$$
\mathcal{H}_{\mathrm{rm}}=\frac{\mathbf{p}^{2}}{2 \mu}+\frac{1}{2} \mu \omega_{0}^{2} r^{2}+\frac{e^{2}}{\kappa r}
$$

where $\mathcal{M}=2 m^{*}, R=|\mathbf{R}|, \mu=m^{*} / 2$, and $r=|\mathbf{r}|$.

The center-of-mass motion associated with the coordinate $\mathbf{R}$ obeys a Schrödinger equation describing the motion of a particle of mass $\mathcal{M}=2 m^{*}$ in a $2 \mathrm{D}$ isotropic harmonic potential of frequency $\omega_{0}$. Here $\omega_{0}$ is the frequency of the original parabolic confinement, i.e., the interelectron repulsion has no baring on the center-of-mass motion.

Using dimensionless polar coordinates $U=R /\left(l_{0} \sqrt{2}\right)$ and $\Theta$, the center-of-mass wave function can be written as $\Xi(U) e^{i M \Theta}$ with the radial part given by

$$
\Xi(U)=C_{N M} U^{|M|} e^{-U^{2} / 2 \mathcal{L}_{0}^{2}} L_{N}^{|M|}\left(U^{2} / \mathcal{L}_{0}^{2}\right),
$$

where $(N, M)$ are the radial and azimuthal (related to the angular momentum) quantum numbers, respectively, $\mathcal{L}_{0}=1 / 2$, the normalization constant $C_{N M}=$ $\left[2 N ! 4^{|M|+1} /(N+|M|) !\right]^{1 / 2}$, and $L_{N}^{|M|}(x)$ are associated Laguerre polynomials.

Since in the exact problem the Coulomb interaction preserves the rotational symmetry, the radial part of the wave function $\Omega(u) e^{i m \theta} / \sqrt{u}$ associated with the relative motion obeys the following one-dimensional
Schrödinger equation [in dimensionless polar coordinates $u=r /\left(l_{0} \sqrt{2}\right)$ and $\left.\theta\right]$,

$$
\frac{\partial^{2} \Omega}{\partial u^{2}}+\left\{\frac{-m^{2}+1 / 4}{u^{2}}-u^{2}-\frac{R_{W} \sqrt{2}}{u}+\frac{\varepsilon}{\hbar \omega_{0} / 2}\right\} \Omega=0 .
$$

The $1 / u$ term results from the interelectron repulsion.

Defining $\widetilde{\mathbf{u}}_{i} \equiv\left(\mathbf{u}_{i}, s_{i}\right)$ (where $s_{i}$ is the spin of the $i$ th electron and $i=1,2$ ), the exact many-body (here twobody) wave function is given by

$$
\begin{aligned}
\Psi\left(\widetilde{\mathbf{u}}_{1}, \widetilde{\mathbf{u}}_{2}\right) & =\Phi\left(\mathbf{u}_{1}, \mathbf{u}_{2}\right) \chi\left(s_{1}, s_{2}\right) \\
& =\frac{1}{2 \pi} \frac{\Omega(u)}{\sqrt{u}} e^{i m \theta} \Xi(U) e^{i M \Theta} \chi\left(s_{1}, s_{2}\right),
\end{aligned}
$$

with $\mathbf{u}_{i}=\mathbf{r}_{i} /\left(l_{0} \sqrt{2}\right)$ and $\chi\left(s_{1}, s_{2}\right)$ is the spin part.

The exact eigenvalues are given by

$$
E_{N M, n m}=\hbar \omega_{0}(2 N+|M|+1)+\varepsilon(n,|m|),
$$

where $\varepsilon(n,|m|)$ are the eigenvalues associated with the relative motion [see Eq. (B5)], $(n, m)$ being the corresponding radial and azimuthal quantum numbers.

\section{Pair correlation, electron density, and conditional probability distribution for the exact and sS-UHF two-electron wave functions}

The bracket notation in Eqs. (3), (4), and (5) imply integrations over both the spatial and spin variables. In the case of the exact wave function $\Psi$ given by Eq. $(\mathrm{B} 6)$, the spin variables separate out, and thus $G(v), n(v)$, and the CPD can be expressed as double integrals over the positions $\left(\mathbf{r}_{1}, \mathbf{r}_{2}\right)$ of the following two-body spatial probability density $W\left(\mathbf{r}_{1}, \mathbf{r}_{2}\right)=\left|\Phi\left(\mathbf{r}_{1}, \mathbf{r}_{2}\right)\right|^{2}$, where $\Phi$ is the spatial part of $\Psi$. In particular, one finds

$$
G(v)=2 \pi \iint \delta\left(\mathbf{r}_{1}-\mathbf{r}_{2}-\mathbf{v}\right) W\left(\mathbf{r}_{1}, \mathbf{r}_{2}\right) d \mathbf{r}_{1} d \mathbf{r}_{2}
$$

for the pair-correlation function,

$$
n(v)=\iint \sum_{i=1}^{2} \delta\left(\mathbf{v}-\mathbf{r}_{i}\right) W\left(\mathbf{r}_{1}, \mathbf{r}_{2}\right) d \mathbf{r}_{1} d \mathbf{r}_{2}
$$

for the electron density, and

$$
\mathcal{P}\left(\mathbf{v} \mid \mathbf{r}_{2}=\mathbf{v}_{0}\right)=\frac{W\left(\mathbf{v}, \mathbf{r}_{2}=\mathbf{v}_{0}\right)}{\int d \mathbf{r}_{1} W\left(\mathbf{r}_{1}, \mathbf{r}_{2}=\mathbf{v}_{0}\right)} .
$$

for the conditional probability distribution.

In the case of the $\mathrm{sS}-\mathrm{UHF}$ singlet ground state, the two electrons occupy two different spatial orbitals $\psi_{1}(\mathbf{r})$ and $\psi_{2}(\mathbf{r})$. Then the corresponding two-body wave function is the following single determinant, 


$$
\begin{aligned}
\Psi^{\mathrm{UHF}}\left(\mathbf{r}_{1}, \mathbf{r}_{2}\right)=\frac{1}{\sqrt{2}} & {\left[\psi_{1}\left(\mathbf{r}_{1}\right) \alpha(1) \psi_{2}\left(\mathbf{r}_{2}\right) \beta(2)\right.} \\
& \left.-\psi_{1}\left(\mathbf{r}_{2}\right) \alpha(2) \psi_{2}\left(\mathbf{r}_{1}\right) \beta(1)\right],
\end{aligned}
$$

where $\alpha$ and $\beta$ denote the spin-up and spin-down spinors, respectively. Integration of $\left|\Psi^{\mathrm{UHF}}\right|^{2}$ over the spin variables yields the following two-body spatial probability density,

$W^{\mathrm{UHF}}\left(\mathbf{r}_{1}, \mathbf{r}_{2}\right)=\frac{1}{2}\left[\left|\psi_{1}\left(\mathbf{r}_{1}\right)\right|^{2}\left|\psi_{2}\left(\mathbf{r}_{2}\right)\right|^{2}+\left|\psi_{1}\left(\mathbf{r}_{2}\right)\right|^{2}\left|\psi_{2}\left(\mathbf{r}_{1}\right)\right|^{2}\right]$.

To calculate the Conditional Probability Distribution in the case of the sS-UHF ground state, one replaces $W$ by $W^{\mathrm{UHF}}$ in Eq. (B10).

${ }^{1}$ M.A. Kastner, Phys. Today 46(1), 24 (1993).

${ }^{2}$ R.C. Ashoori, Nature 379, 413 (1996).

${ }^{3}$ S. Tarucha, D.G. Austing, T. Honda, R.J. van der Hage, and L.P. Kouwenhoven, Phys. Rev. Lett. 77, 3613 (1996).

${ }^{4}$ E.U. Condon and G.H. Shortley, The theory of atomic spectra (Cambridge Univ. Press, London, 1935).

${ }^{5}$ D.R. Hartree, Proc. Camb. Phil. Soc. 24, 89 (1928).

${ }^{6}$ The failure of Bohr-type models, based on the orbiting of spatially correlated electrons in conjunction with the Bohr-Sommerfeld quantization rule, to yield a reasonable estimate of the ground state of the $\mathrm{He}$ atom signaled a looming crisis in physics in the 1920's, which Bohr himself, as well as others, had been keenly aware of, as summarized succintly by Sommerfeld: "All attempts made hitherto to solve the problem of the neutral helium atom have proved to be unsuccessful" [A. Sommerfeld, Rev. Sci. Inst. 7, 509 (1923)]; see also the 10th chapter entitled "It was the Spring of hope, it was the Winter of despair" in A. Pais, Niels-Bohr's Times (Clarendon, Oxford, 1991), the review by J.H. Van Vleck, Phil. Mag. 44, 842 (1922), and M. Born, The Mechanics of the Atom (Ungar, New York, 1927). While, since, numerical solutions of the twoelectron Schrödinger equation provided a quantitative resolution to the problem, the first successful semiclassical treatment of the three-body Coulomb system awaited till 1980, see J.G. Leopold and I.C. Percival, J. Phys. B 13, 1037 (1980); see also D. Wintgen, K. Richter and G. Tanner, Chaos 2, 19 (1992). Furthermore, based on rather general group-theoretical arguments arising from the observation of hierarchies with lower symmetry in the excited spectra, and motivated by ideas originating in nuclear-physics spectroscopy, it has been discovered in the late 1970's and early 1980's that electron correlations in doubly excited He lead to quantization of the spectrum much like in a linear triatomic molecule, $e-\mathrm{He}^{2+}-e$. This molecular picture, with near rigidity and separability, results in "infinite sequences of vibrational levels, on each of which is built an infinite sequence of rotational levels", see M.E. Kellman and
D.R. Herrick, Phys. Rev. A 22, 1536 (1980); see also G.S. Ezra and R.S. Berry, Phys. Rev. A 28, 1974 (1983), and S. Watanabe and C.D. Lin, Phys. Rev. A 34, 823 (1986). Interestingly, as in the aforementioned semiclassical treatments, the collinear configuration plays a special role in the molecule-like model, serving perhaps as "partial vindication" of the geometry considered originally by Bohr.

${ }^{7} \AA$ A. Bohr and B.R. Mottelson, Nuclear structure (Benjamin, Reading, MA, 1975).

${ }^{8}$ W. Nazarewicz, Nucl. Phys. A 574, 27c (1994).

${ }^{9}$ C. Yannouleas and U. Landman, Phys. Rev. B 51, 1902 (1995).

${ }^{10}$ W. Ekardt, Metal clusters Ch. 1 \& Ch. 4 (Wiley, New York, 1999).

${ }^{11}$ In modeling of practical "on-off" (i.e., two-state) devices, one will undoubtedly have to take into account additional potential terms in the many-body hamiltonian, such as those associated with image charges resulting from interfaces between different dielectric materials. The feasibility of constructing nanoscale logic cells via controlled manipulation of the antipodal arrangements of two electrons in a larger device made out of four dots arranged in a square to form a quantum-dot supermolecule is currently under intense experimental investigation, see I. Amlani, A.O. Orlov, G. Toth, G.H. Bernstein, C.S. Lent, and G.L. Snider, Science 284, 289 (1999).

${ }^{12}$ E. Wigner, Phys. Rev. 46, 1002 (1934).

13 P.A. Maksym, Phys. Rev. B 53, 10871 (1996).

${ }^{14}$ H.-M. Müller and S.E. Koonin, Phys. Rev. B 54, 14532 (1996).

${ }^{15}$ R. Egger, W. Häusler, C.H. Mak, and H. Grabert, Phys. Rev. Lett. 82, 3320 (1999).

${ }^{16}$ C. Yannouleas and U. Landman, Phys. Rev. Lett. 82, 5325 (1999).

17 P.W. Anderson, Basic notions of condensed matter physics (Addison-Wesley, Reading, MA, 1984).

18 D.J. Thouless, The quantum mechanics of many-body systems (Academic Press, New York, 1972).

${ }^{19}$ H. Fukutome, Int. J. Quant. Chem. 20, 955 (1981).

${ }^{20}$ For studies pertaining to the arrangement of classical pointcharges in a harmonic confinement, see Yu.E. Lozovik, Usp. Fiz. Nauk 153, 356 (1987) [Sov. Phys. Usp. 30, 912 (1987)] and V.M. Bedanov and F.M. Peeters, Phys. Rev. B 49, 2667 (1994).

21 That $R_{W}$ is the only relevant parameter in the formation of a WM in a clean QD can be seen immediately from the exact Schrödinger equation describing the relative motion of two interacting electrons at $B=0$ in a parabolic confinement (Appendix B). Indeed, this equation when expressed in dimensionless variables as in Eq. (B5) contains $R_{W}$ as the sole free parameter. The corresponding center-of-mass motion is independent of the interelectron repulsion. For how to extract information about the Wigner molecule from the exact two-body solution, see section IV.

${ }^{22}$ B. Tanatar and D.M. Ceperley, Phys. Rev. B 39, 5005 (1989).

${ }^{23}$ In all calculations, we used $g^{*}(\mathrm{GaAs})=-0.44, m^{*}(\mathrm{GaAs})=$ $0.067 m_{e}$, and $\hbar \omega_{0}=5 \mathrm{meV}$, with $R_{W}=2.39$ (that is $\kappa=$ $8)$; the same value of $R_{W}$ is obtained by using $\kappa(\mathrm{GaAs})=$ 12.9 and $\hbar \omega_{0}=1.9 \mathrm{meV}$, yielding the similar results given 
here, but with different absolute energy values.

${ }^{24}$ M. Wagner, U. Merkt, and A.V. Chaplik, Phys. Rev. B 45, 1951 (1992).

${ }^{25}$ D. Pfannkuche, V. Gudmundsson, and P.A. Maksym, Phys. Rev. B 47, 2244 (1993).

${ }^{26}$ M. Taut, J. Phys. A 27, 1045 (1994).

27 M. Taut, Phys. Rev. A 48, 3561 (1993).

${ }^{28}$ M. Rontani, F. Rossi, F. Manghi, and E. Molinari, Phys. Rev. B 59, 10165 (1999).

${ }^{29}$ Here we differentiate between the exact solution of the hamiltonian specified by Eqs. (1) and (2) from "exact diagonalization methods" of Hubbard-type hamiltonians used as models of QD's [see e.g., R. Berkovits, Phys. Rev. Lett. 81, 2128 (1998)].

30 The sS-UHF equations were solved using the Pople-NesbetRoothaan formalism, see p. 214 in Ref. 31; see also J.A. Pople and R.K. Nesbet, J. Chem. Phys. 22, 571 (1954). Apart from Ref. 16, the only previous use of a Space-UHF method in QD's was in an investigation (see Ref. 14) of WM's but only for fully polarized QD's (i.e., under high magnetic fields where the spin unrestriction is not at play).

${ }^{31}$ A. Szabo and N.S. Ostlund, Modern quantum chemistry (McGraw-Hill, New York, 1989).

32 E.K.U. Gross, E. Runge, and O. Heinonen, Many-particle theory (Adam Hilger, Bristol, 1991) ch. 8.

${ }^{33}$ From the point of view of the terminology adopted here, the simple designation Hartree-Fock (HF) in the literature most often refers to the RHF, in particular in atomic physics and the physics of the homogeneous electron gas. In nuclear physics, however, the simple designation HF most often refers to a space (S)-UHF.

${ }^{34}$ see the article by Ezra and Berry in Ref. 6; see also R.S. Berry in Structure and dynamics of atoms and molecules: Conceptual trends, edited by J.L. Calais and E.S. Kryachko (Kluwer Academic, Dordrecht, 1995) p. 155, and references therein.

35 The orientational degeneracy of the sS-UHF solutions describing an electron molecule in a cirularly symmetric QD is a residue of the rotational invariance of the exact hamiltonian and naturally it suggests the possible emergence of a ro-vibrational spectrum associated with the excitations of the electron molecule. Depending on the value of the parameter $R_{W}$, the electron molecule may exhibit different degrees of "rigidity", with electron molecules of high rigidity (forming for large $R_{W}$ ) exhibiting well-developed ro-vibrational bands; for the value of $R_{W}=2.39$ used by us here, the electron molecule is rather "floppy" [C. Yannouleas and U. Landman, cond-mat/0003245. Such rovibrational spectra can be described by several theoretical approaches, for example: (i) at a more phenomenological level, by a quantization of the collective classical motions associated with the molecule, such as rotations, and bending and stretching vibrations [see Kellman and Herrick in Ref. 6 and Maksym in Ref. 13]; (ii) at a microscopic level, by projecting the symmetry-broken HF determinant onto multi-determinental wave functions with good angular momenta, thus restoring the symmetry of the exact hamiltonian; see P. Ring, and P. Schuck, The nuclear manybody problem, Ch. 11 (Springer-Verlag, New York, 1980) and P.-O. Löwdin, in Quantum theory of atoms, molecules, and the solid state, Edited by P.-O. Löwdin, pp. $601-623$ (Academic Press, New York, 1966). Furthermore, starting from an exact solution for the electrons in the QD, rovibrational characteristics can be obtained from the manybody eigenvalue spectra evaluated for different total angular momenta and spins in conjunction with an analysis of the conditional-probability densities [C. Yannouleas and U. Landman, cond-mat/0003245. We note that the discrete ro-vibrational collective spectra associated with symmetrybreaking in a QD may be viewed as finite analogs to the Goldstone modes accompanying symmetry breaking transitions in extended media (see Ref. 17).

${ }^{36}$ H.A. Jahn and E. Teller, Proc. Roy. Soc. London Ser. A 161, 220 (1937).

${ }^{37}$ Recall that for an electron moving in a $2 \mathrm{D}$ circularly symmetric potential the wave functions are given (in polar coordinates) by $\Psi_{n_{r}, m}(r, \theta)=R\left(r ; n_{r},|m|\right) \exp (i m \theta)$ with corresponding energies $\mathcal{E}\left(n_{r},|m|\right)$, where $n_{r}$ is the number of radial nodes and $m$ is the angular momentum quantum number, with $m=0, \pm 1, \pm 2, \ldots$ In the JT framework, the second electron of the (open-shell) triplet state of the $2 e$ QD occupies an orbital formed as a linear combination of the $n_{r}=0, m= \pm 1$ states (denoted by $p_{+}$and $p_{-}$), i.e., $p_{x} \propto p_{+}+p_{-}$or $p_{y} \propto p_{+}-p_{-}$, with the one of lower energy being selected through the self-consistent distortion of the central mean field. As a consequence, the orbitals of both electrons in the $2 e \mathrm{QD}$ exhibit noncircular symmetry. The energy gain due to the JT distortion depends on the strength of the confinement potential, and it increases for weaker confinements. In natural atoms, the spontaneous JT effect is fairly small and perturbative methods are usually adequate; see Ch. III.2 in Ref. 18.

38 This cancellation of crystallization due to a strongattractive-impurity effect is illustrated in Fig. 4(d) where the attractive impurity is able to capture two of the eight electrons in the dot. The two captured electrons form a singlet subunit and their orbitals are clearly circularly symmetric (see Appendix A below).

39 The effective confinement frequency $\Omega_{\text {eff }}=\left(\omega_{0}^{2}+\omega_{c}^{2} / 4\right)^{1 / 2}$ in the Darwin-Fock model of a $2 \mathrm{D}$ harmonically-confined electron in the presence of a magnetic field $B$ (where $\omega_{0}$ is the frequency of the harmonic potential and $\omega_{c}=e B / m^{*} c$ is the cyclotron frequency) increases as a function of the magnetic field. In a magnetic field, the energy of a harmonically confined electron is given in the Darwin-Fock model by $E_{n_{r}, m}=\left(2 n_{r}+|m|+1\right) \hbar \Omega_{e f f}-m \hbar \omega_{c} / 2$.

${ }^{40}$ Such JT-like electron molecules have been called Wigner molecules in previous publications treating fully polarized QD's (Refs. 13 and 14). However, this naming is not appropriate since their formation is not driven by the dominance of the interelectron repulsion (over the zero-point energy), which for large magnetic fields can be treated perturbatively (Refs. 24 and 26.).

41 Å. Bohr and B.R. Mottelson, Mat. Fys. Medd. Dan. Vidensk. Selsk. 27 (No. 16) (1953).

${ }^{42}$ S.G. Nilsson, Mat. Fys. Medd. Dan. Vidensk. Selsk. 29 (No. 16) (1955).

${ }^{43}$ V.M. Strutinsky, Nucl. Phys. A95, 420 (1967).

${ }^{44}$ For an early review of the application of Hartree-Fock methods to the description of open-shell, deformed nuclei, 
see G. Ripka, Adv. Nucl. Phys. 1, 183 (1968).

${ }^{45}$ R.E. Peierls and J. Yoccoz, Proc. Phys. Soc. (London) A70, 381 (1957).

${ }^{46}$ R.E. Peierls and D.J. Thouless, Nucl. Phys. 38, 154 (1962).

47 The concept of restoration of symmetry is not restricted to spatial symmetry, but includes also restoration of spin symmetry (see the paper by Löwdin in Ref. 35 .

${ }^{48}$ G.E. Brown, Unified theory of models and forces (NorthHolland, Amsterdam, 1967) p. 64.

${ }^{49}$ For the purely parabolic confinement considered here, it turns out that the pair-correlation function reduces27 to the modulus square of the radial part of the wave function (see Appendix B) describing the relative motion, i.e., $G(v)=$ $\Omega^{2}(v) / v$.

50 See p. 620 of the article by Löwdin mentioned in Ref. 35 (in this article the RHF is referred to as conventional HartreeFock scheme); this definition was also followed in Ref. 25, where a RHF solution was contrasted to the exact groundstate solution for two interacting electrons.

${ }^{51}$ C. Yannouleas and U. Landman, to be published.

FIG. 1. Total electron densities (left frame in each pannel) and contours of modulus square of the individual orbitals (right frames) for the clean (Thomson) $2 e$ TQD at $B=0$, in the presence of two attractive $\left(\mathcal{Q}_{1}=\mathcal{Q}_{2}=-1 e\right)$ impurities whose projected positions on the QD plane $(x y)$ are denoted by filled circles. (a) The symmetry-adapted (RHF) singlet $(\mathcal{S})$. The energy of the SA singlet is higher by $1.34 \mathrm{meV}$ than that of the corresponding triplet, manifesting a shortcoming of the RHF method. (b) The sS-UHF Wigner-molecule singlet, orientationally pinned along the inter-impurity axis and exhibiting localized orbitals, one to the left and the other to the right of the QD middle; note the lack of reflection symmetry of the individual orbitals about the mirror $(y z)$ plane normal to the inter-impurity axis and passing through the QD's center. When the WM singlet is rotated by $\pi / 2$, the energy of the system rises by $0.58 \mathrm{meV}$. (c) The triplet $(\mathcal{T})$ state with an elliptically deformed $s$-like and a $p_{x}$-like orbital, orientationally pinned by the impurities. The energy of the symmetry-broken singlet in (b) is lower by $0.28 \mathrm{meV}$ than that of the triplet in (c), compared to $0.43 \mathrm{meV}$ for the TQD without pinning impurities. Distances are in $\mathrm{nm}$ and the electron densities in $10^{-4} \mathrm{~nm}^{-2}$. The parameters characterizing the QD shown here are those given in Ref. 23.
FIG. 2. (a-d) Energetics and individual orbitals (modulus square) from sS-UHF calculations for the $2 e$ (Rutherford) RQD, with a central impurity $\mathcal{Q}=-2 e$ at $(0,0,10) \mathrm{nm}$. Energies (in meV) of the $\mathcal{S}$ and $\mathcal{T}$ states versus $B$ (in Tesla), crossing at $B_{c}=2.8 \mathrm{~T}$ (marked by an arrow), are shown in (a). An expanded view of the energy of the $\mathcal{T}$ state for small fields, exhibiting a transition from the spontaneous JT regime to a circular symmetric state at $B>B_{1}\left(B_{1}=0.2 \mathrm{~T}\right)$, is shown in the inset; included also is the energy of the SA solution (dashed curve). At $B=0$, contours of the orbitals of the two electrons for the WM singlet are shown (superimposed on each other) in (b), with one of the orbitals depicted by a solid line and the other by a dashed line. The orbitals for the spontaneously JT-distorted triplet at $B=0$ are shown in the two panels of (c). For the singlet, the energy gain due to $\mathrm{SB}$ (that is lowering of the total energy with respect to the symmetry-adapted RHF state) is $0.39 \mathrm{meV}$ and the energy gain due to the JT-distortion of the triplet is $0.07 \mathrm{meV}$; the energy difference between the $\mathcal{T}$ and $\mathcal{S}$ states is $2.01 \mathrm{meV}$. The transition to a circular symmetric triplet is illustrated for $B=0.6 \mathrm{~T}$ in (d). (e) Individual orbitals for the symmetry-broken ground-state triplet of the clean (Thomson) $2 e$ TQD at $B=10 \mathrm{~T}$. Under the same conditions, the ground state triplet in the $2 e$ RQD remains circularly symmetric. Distances in $\mathrm{nm}$ and orbital densities in $10^{-4} \mathrm{~nm}^{-2}$. Note the different length scales of the $(x, y)$ axes in (b-e) compared to those in Fig. 1. The parameters characterizing the QD's shown here are those given in Ref. 23.

FIG. 3. Ground-state results for a clean 2e QD with the parameters given in Ref. 23 and $B=0$; in all cases the ground state is a singlet. (a-c) correspond to the exact solution. (a) The pair correlation function $[G(v)$, see Eq. (3) $]$ plotted versus $v$ exhibiting a well developed depression at $v=0$, with a mean electron-to-electron separation of $2 r_{0}=22.92 \mathrm{~nm}$. (b) The electron conditional probability distribution [CPD, see Eq. (5)] with $\mathbf{v}_{0}=\left(r_{0}, 0\right)$ (denoted by a cross), showing formation of a $2 \mathrm{e}$ electron molecule. (c) The electron density [ED, see Eq. (4)], reflecting the conservation of circular symmetry by the exact two-electron solution. The results displayed in (d) and (e) correspond to calculations using the sS-UHF method. Spontaneous symmetry breaking leading to formation of a 2e-molecule (with a bond length of $2 r_{0}^{\prime}=28.16$ $\mathrm{nm}$ ) is exhibited by the sS-UHF electron density shown in (d). (e) Such an electron molecule is further reflected in the sS-UHF CPD with $\mathbf{v}_{0}=\left(r_{0}^{\prime}, 0\right)$ (denoted by a cross). (f) The CPD with $\mathbf{v}_{0}=\left(r_{0}^{\prime}, 0\right)$ (denoted by a cross) corresponding to the restricted Hartree-Fock (RHF) ground state (i.e., the symmetry-adapted solution) exhibits, as expected, no symmetry-breaking signature. Lengths are in $\mathrm{nm}$ and density functions $\left[G(v)\right.$, ED's, and CPD's] are in units of $10^{-4} \mathrm{~nm}^{-2}$. 
FIG. 4. Electron densities obtained via sS-UHF calculations of a $\mathrm{QD}$ containing 8 electrons at $B=0$ in the presence of a central [located at $(0,0,0)$ in $(\mathrm{a}-\mathrm{c})]$ and an off-centerd [located at $(20,0,0) \mathrm{nm}$ in (d)] hydrogenic impurity. The parameters characterizing the QD shown here are those given in Ref. 23 and the charge $\mathcal{Q}$ of the impurity is as marked on the figure. All the cases correspond to zero total spin, i.e., $4 \mathrm{e}$ with spin up and $4 \mathrm{e}$ with spin down. For each of the cases, we observe formation of a Wigner molecule, with its structure dependent on the polarity ( $\operatorname{sign})$, magnitude and location of the impuriry. (a) A repulsive central impurity $(\mathcal{Q}=+1 e)$, resulting in an $8 \mathrm{e} \mathrm{WM}$ with a ring structure and an empty-electron central region, denoted as $(0,8)$. (b) Slightly attractive central impurity $(\mathcal{Q}=-0.2 e)$ leading to formation of an $(1,7)$ two-ring WM. (c) A stronger attractive central impurity $(\mathcal{Q}=-0.5 e)$ associated with a $(2,6)$ WM. (d) An off-center attractive impurity $(\mathcal{Q}=-1 e)$, resulting in a distorted 6e WM with two electrons trapped by the impurity. Lengths in $\mathrm{nm}$ and electron densities in units of $10^{-3}$ $\mathrm{nm}^{-2}$. 
This figure "qdh1j.gif" is available in "gif" format from: http://arxiv.org/ps/cond-mat/0002364v2 
This figure "qdh2j.gif" is available in "gif" format from: http://arxiv.org/ps/cond-mat/0002364v2 
This figure "qdh3j.gif" is available in "gif" format from: http://arxiv.org/ps/cond-mat/0002364v2 
This figure "qdh4j.gif" is available in "gif" format from: http://arxiv.org/ps/cond-mat/0002364v2 